\title{
FINITE ELEMENT LEAST-SQUARES METHODS FOR A COMPRESSIBLE STOKES SYSTEM
}

\author{
KEEHWAN KIM, SANGDONG KIM, and SANGSIK SHIN
}

Received 25 April 2002

\begin{abstract}
The least-squares functional related to a vorticity variable or a velocity flux variable is considered for two-dimensional compressible Stokes equations. We show ellipticity and continuity in an appropriate product norm for each functional.
\end{abstract}

2000 Mathematics Subject Classification: 65F10, 65F30.

1. Introduction. Let $\Omega$ be a convex polygonal domain in $\mathbb{R}^{2}$. Consider the stationary compressible Stokes equations with zero boundary conditions for the velocity $\mathbf{u}=$ $\left(u_{1}, u_{2}\right)^{t}$ and pressure $p$ as follows:

$$
\begin{gathered}
-\mu \Delta \mathbf{u}+\nabla p=\mathbf{f} \quad \text { in } \Omega, \\
\nabla \cdot \mathbf{u}+\boldsymbol{\beta} \cdot \nabla p=g \quad \text { in } \Omega, \\
\mathbf{u}=\mathbf{0} \quad \text { on } \partial \Omega,
\end{gathered}
$$

where the symbols $\Delta, \nabla$, and $\nabla \cdot$ stand for the Laplacian, gradient, and divergence operators, respectively ( $\Delta \mathbf{u}$ is the vector of components $\Delta u_{i}$ ); the number $\mu$ is a viscous constant; $\mathbf{f}$ is a given vector function; $\boldsymbol{\beta}=(U, V)^{t}$ is a given $C^{1}$ function. The system (1.1) may be obtained by linearizing the steady-state barotropic compressible viscous Navier-Stokes equations without an ambient flow (see $[8,9]$ for more detail). Since the continuity equation is of hyperbolic type containing a convective derivative of $p$, we further assume that the boundary condition for the pressure is given on the inlet of the boundary where the characteristic function $\boldsymbol{\beta}$ points into $\Omega$, that is,

$$
p=0 \quad \text { on } \Gamma_{\text {in }},
$$

where $\Gamma_{\text {in }}=\{(x, y) \in \partial \Omega \mid \boldsymbol{\beta} \cdot \mathbf{n}<0\}$ with the outward unit normal $\mathbf{n}$ to $\partial \Omega$. Hence the boundary $\partial \Omega$ consists of $\Gamma_{\text {in }}$ and $\Gamma_{\text {out }}$ where $\Gamma_{\text {out }}=\{(x, y) \mid \boldsymbol{\beta} \cdot \mathbf{n} \geq 0\}$. There was a study on a mixed finite element theory for a compressible Stokes system (see, e.g., [8]), but there are a few trials dealing with a compressible Stokes system like (1.1) using leastsquares method. Some papers focused on a $H^{-1}$ least-squares method (see, e.g., [6, 9]). Least-squares approach was developed for the incompressible Stokes and Navier-Stokes equations in [1, 2, 7]. The purpose of this paper is to apply the philosophy of firstorder system least-squares (FOSLS) methodology developed in [5] to a compressible stationary Stokes system. We consider two basic first-order systems. The first one is induced by a vorticity variable, and the second one is induced by a velocity flux variable 
which is further extended to the system's associated curl and trace equations. This extended system is not a system of first order but a mixture system of first- and secondorder equations due to the continuity equation $\nabla \cdot \mathbf{u}+\boldsymbol{\beta} \cdot \nabla p=g$. In order to provide ellipticity for each functional, we assume the $H^{1}$ and $H^{2}$ regularity assumptions for the compressible Stokes equations. As usual in FOSLS approach, we first show that the $H^{-1}$ and $L^{2}$ FOSLS functional is elliptic in the product norm $\|w\|+\|\mathbf{u}\|_{1}+\|q\|+\|p\|_{0, \boldsymbol{\beta}}$ for the functional involving vorticity variable and $\|\mathbf{U}\|+\|\mathbf{u}\|_{1}+\|p\|$ for the functional involving flux variable. We also show that the extended functional related to velocity flux variable is elliptic in the product norm $\|\mathbf{U}\|_{1}+\|\mathbf{u}\|_{1}+\|p\|_{1, \boldsymbol{\beta}}$. Then we provide the error estimates for using finite element methods. The outline of the paper is as follows. In Section 2, we discuss least-squares system and other preliminaries. The continuity and ellipticity of least-squares functionals are discussed in Section 3. These can be done by employing regularity estimates for (1.1). The finite element approximations are briefly discussed in Section 4.

\section{Least-squares system for compressible Stokes equations, and other prelimi-} naries. For the development of least-squares theory, we will adopt the notation introduced in [5] and introduce the necessary definitions in this section. A new independent variable related to the 4 -vector function of gradients of the displacement vectors, $u_{i}$, $i=1,2$ will be given. It will be convenient to view the original $n$-vector functions as column vectors and the new 4 -vector functions as either block column vectors or matrices. The velocity variable $\mathbf{u}=\left(u_{1}, u_{2}\right)^{t}$ is a column vector with scalar components $u_{i}$, so that the gradient $\nabla \mathbf{u}^{t}$ is a matrix with columns $\nabla u_{i}$. For a function $\mathbf{U}$ with 2-vector components $\mathbf{U}_{i}$

$$
\mathbf{U}=\nabla \mathbf{u}^{t}=\left(\mathbf{U}_{1}, \mathbf{U}_{2}\right)=\left(U_{i j}\right)_{2 \times 2},
$$

which is a matrix with entries $U_{i j}=\partial u_{j} / \partial x_{i}, 1 \leq i, j \leq 2$. Then we can define the trace operator tr as

$$
\operatorname{tr} \mathbf{U}=\sum_{i=1}^{n} U_{i i} .
$$

Let, for $\mathbf{v} \in L^{2}(\Omega)^{2}$,

$$
\begin{gathered}
\nabla \times \mathbf{v}:=\operatorname{curl} \mathbf{v}=\frac{\partial v_{2}}{\partial x}-\frac{\partial v_{1}}{\partial y}, \quad \nabla \cdot \mathbf{v}=\frac{\partial v_{1}}{\partial x}+\frac{\partial v_{2}}{\partial y} \\
\nabla^{\perp} \mathbf{v}^{t}=\left(\nabla^{\perp} v_{1}, \nabla^{\perp} v_{2}\right)=\left(\begin{array}{cc}
\partial_{y} v_{1} & \partial_{y} v_{2} \\
-\partial_{x} v_{1} & -\partial_{x} v_{2}
\end{array}\right), \\
\mathbf{n} \times \mathbf{v}=-n_{2} v_{1}+n_{1} v_{2} .
\end{gathered}
$$

Define the curl as

$$
\nabla \times \mathbf{U}=\left(\nabla \times \mathbf{U}_{1}, \nabla \times \mathbf{U}_{2}\right),
$$


and the divergence as

$$
(\nabla \cdot \mathbf{U})^{t}=\left(\nabla \cdot \mathbf{U}_{1}, \nabla \cdot \mathbf{U}_{2}\right)^{t}
$$

We also define the tangential operator $\mathbf{n} \times$ componentwise

$$
\mathbf{n} \times \mathbf{U}=\left(\mathbf{n} \times \mathbf{U}_{1}, \mathbf{n} \times \mathbf{U}_{2}\right) .
$$

The inner products and norms on the block column vector functions are defined in the natural componentwise way; for example,

$$
\|\mathbf{U}\|^{2}=\sum_{i=1}^{2}\left\|\mathbf{U}_{i}\right\|^{2}=\sum_{i, j=1}^{2}\left\|U_{i j}\right\|^{2} .
$$

We use standard notations and definitions for the Sobolev spaces $H^{s}(\Omega)^{n}$, associated inner products $(\cdot, \cdot)_{s}$, and respective norms $\|\cdot\|_{s}, s \geq 0$. When $s=0, H^{0}(\Omega)^{n}$ is the usual $L^{2}(\Omega)^{n}$, in which case the norm and inner product will be denoted by $\|\cdot\|_{0}=\|\cdot\|$ and $(\cdot, \cdot)$, respectively. The space $H_{0}^{s}(\Omega)$ is the set of functions in $H^{s}(\Omega)$ vanishing on the boundaries. From now on, we will omit the superscript $n$ and $\Omega$ if the dependence of vector norms on dimension is clear by context. We use $H_{0}^{-1}(\Omega)$ to denote the dual spaces of $H_{0}^{1}(\Omega)$ with norm defined by

$$
\|\phi\|_{-1}=\sup _{\psi \in H_{0}^{1}(\Omega)} \frac{(\phi, \psi)}{\|\psi\|_{1}} .
$$

Define the product spaces $H_{0}^{s}(\Omega)^{2}$ and $L^{2}(\Omega)^{2}$ in usual way with standard product norms. Let

$$
H(\operatorname{div} ; \Omega)=\left\{\mathbf{v} \in L^{2}(\Omega)^{2}: \nabla \cdot \mathbf{v} \in L^{2}(\Omega)\right\}
$$

Define a space

$$
Q_{k}(\Omega)=\left\{q \in L^{2}(\Omega):\left(\|q\|_{k}^{2}+\|\boldsymbol{\beta} \cdot \nabla q\|_{k}^{2}\right)^{1 / 2}<\infty\right\}
$$

where $k$ is either 1 or 0 , which is a Hilbert space with norm

$$
\|q\|_{k, \boldsymbol{\beta}}=\left(\|q\|_{k}^{2}+\|\boldsymbol{\beta} \cdot \nabla q\|_{k}^{2}\right)^{1 / 2} .
$$

We frequently use the notation constant $C_{\Omega}$ to denote that it depends on $\Omega$ only, but it may be a different constant. If a constant depends on another variable, we specify it in each place. Throughout this paper, we assume the following regularity.

AssumPtion 1. Assume that $\mu$ and $\boldsymbol{\beta}$ are such that (1.1) has a unique solution which satisfies the following a priori estimate:

$$
\left\|\nabla \mathbf{u}^{t}\right\|_{k}^{2}+\|p\|_{k}^{2} \leq C_{0}(\mu, \Omega)\left(\|-\mu \Delta \mathbf{u}+\nabla p\|_{k-1}^{2}+\|\nabla \cdot \mathbf{u}+\boldsymbol{\beta} \cdot \nabla p\|_{k}^{2}\right),
$$

where $k$ is either 0 or $1 ; C_{0}:=C_{0}(\mu, \Omega)$ is a constant depending on $\mu, \boldsymbol{\beta}$, and $\Omega$. Note that one may find (2.12) for $k=1$ in [10, Theorem 1.3] for $\boldsymbol{\beta}=(1,0)^{t}$ and one may get (2.12) 
for $k=0$ by following the arguments in [10, Section 3]. In fact, using triangle inequality and the assumption (2.12), one may get the improved a priori estimates:

$$
\left\|\nabla \mathbf{u}^{t}\right\|_{k}^{2}+\|p\|_{k, \boldsymbol{\beta}}^{2} \leq C_{0}(\mu, \Omega)\left(\|-\mu \Delta \mathbf{u}+\nabla p\|_{k-1}^{2}+\|\nabla \cdot \mathbf{u}+\boldsymbol{\beta} \cdot \nabla p\|_{k}^{2}\right),
$$

where $k$ is 1 or 0 and $C_{0}:=C_{0}(\mu, \Omega)$ is a constant depending on $\mu, \boldsymbol{\beta}$, and $\Omega$.

2.1. Velocity-vorticity-pressure formulation. Note that

$$
\nabla^{\perp}(\nabla \times \mathbf{u})=-\Delta \mathbf{u}+\nabla(\nabla \cdot \mathbf{u}) .
$$

As in [4] for Stokes equations, introducing the vorticity variable $w=\nabla \times \mathbf{u}$, the first equation of the compressible Stokes equations (1.1) using the second equation of (1.1) is

$$
\mu \nabla^{\perp} w-\mu \nabla \cdot q+\nabla p=\mathbf{f} .
$$

By setting $q=\nabla \cdot \mathbf{u}$, the equivalent first-order system is now

$$
\begin{gathered}
w-\nabla \times \mathbf{u}=0 \quad \text { in } \Omega, \\
q-\nabla \cdot \mathbf{u}=0 \quad \text { in } \Omega, \\
\mu \nabla^{\perp} w-\mu \nabla q+\nabla p=\mathbf{f} \quad \text { in } \Omega, \\
q+\boldsymbol{\beta} \cdot \nabla p=g \quad \text { in } \Omega, \\
\mathbf{u}=\mathbf{0} \quad \text { on } \partial \Omega, \\
p=0 \quad \text { on } \Gamma_{\text {in }} .
\end{gathered}
$$

2.2. Velocity-flux-pressure formulation. As in [5] for Stokes equations, introducing the velocity flux variable $\mathbf{U}=\nabla \mathbf{u}^{t}$, the compressible Stokes equations (1.1) may be written as the following equivalent first-order system:

$$
\begin{gathered}
\mathbf{U}-\nabla \mathbf{u}^{t}=\mathbf{0} \quad \text { in } \Omega, \\
-\mu(\nabla \cdot \mathbf{U})^{t}+\nabla p=\mathbf{f} \quad \text { in } \Omega, \\
\nabla \cdot \mathbf{u}+\boldsymbol{\beta} \cdot \nabla p=g \quad \text { in } \Omega, \\
\mathbf{u}=\mathbf{0} \quad \text { on } \partial \Omega, \\
p=0 \quad \text { on } \Gamma_{\text {in }} .
\end{gathered}
$$

We consider the following extended equivalent system for (2.17):

$$
\begin{gathered}
\mathbf{U}-\nabla \mathbf{u}^{t}=\mathbf{0} \quad \text { in } \Omega, \\
-\mu(\nabla \cdot \mathbf{U})^{t}+\nabla p=\mathbf{f} \quad \text { in } \Omega, \\
\nabla \cdot \mathbf{u}+\boldsymbol{\beta} \nabla p=g \quad \text { in } \Omega, \\
\nabla \times \mathbf{U}=\mathbf{0} \quad \text { in } \Omega, \\
\nabla(\operatorname{tr} \mathbf{U})+\nabla(\boldsymbol{\beta} \cdot \nabla p)=\nabla g \quad \text { in } \Omega, \\
\mathbf{u}=\mathbf{0} \quad \text { on } \partial \Omega, \\
\mathbf{n} \times \mathbf{U}=\mathbf{0} \quad \text { on } \partial \Omega, \\
p=0 \quad \text { on } \Gamma_{\text {in }} .
\end{gathered}
$$


3. Least-squares functionals. The main objective in this section is to establish ellipticity and continuity of least-squares functionals based on (2.16), (2.17), and (2.18) in appropriate Sobolev spaces.

3.1. Velocity, vorticity, and pressure. The first-order least-squares functional corresponding to (2.16) is

$$
\begin{aligned}
G_{0}(w, \mathbf{u}, q, p ; \mathbf{f}, g)= & \left\|\mu \nabla^{\perp} w-\mu \nabla q+\nabla p-\mathbf{f}\right\|_{-1,0}^{2} \\
& +\|q+\boldsymbol{\beta} \cdot \nabla p-g\|^{2}+\|w-\nabla \times \mathbf{u}\|^{2}+\|q-\nabla \cdot \mathbf{u}\|^{2}
\end{aligned}
$$

Define

$$
M_{0}(w, \mathbf{u}, q, p)=\|w\|^{2}+\|\mathbf{u}\|_{1}^{2}+\|q\|^{2}+\|p\|_{0, \boldsymbol{\beta}}^{2}
$$

and let

$$
\mathscr{V}_{0}=L^{2}(\Omega) \times H_{0}^{1}(\Omega)^{2} \times L^{2}(\Omega) \times Q_{0}(\Omega)
$$

The FOSLS variational problem for the compressible Stokes equations corresponding to (2.16) is to minimize the quadratic functional $G_{0}$ over $\mathscr{V}_{0}$ : find $(w, \mathbf{u}, q, p) \in \mathscr{V}_{0}$ such that

$$
G_{0}(w, \mathbf{u}, q, p ; \mathbf{f}, \mathfrak{g})=\inf _{(z, \mathbf{v}, r, s) \in \mathcal{V}_{0}} G_{0}(z, \mathbf{v}, r, s ; \mathbf{f}, \mathfrak{g}) .
$$

THEOREM 3.1. Under the assumption (2.12), there are two positive constants $c$ and $C$, dependent on $\delta$ and $\Omega$, such that for all $(w, \mathbf{u}, q, p) \in \mathscr{V}_{0}$,

$$
c M_{0}(w, \mathbf{u}, q, p) \leq G_{0}(w, \mathbf{u}, q, p ; \mathbf{0}, 0) \leq C M_{0}(w, \mathbf{u}, q, p) .
$$

Proof. Upper bound in (3.5) is a simple consequence of the triangle inequality and Cauchy-Schwarz inequality. For any $(w, \mathbf{u}, q, p) \in \mathscr{V}_{0}$, using (2.13), triangle inequality, and $(\cdot)$, we have

$$
\begin{aligned}
\left\|\nabla \mathbf{u}^{t}\right\|^{2}+\|p\|_{0, \boldsymbol{\beta}}^{2} \leq & C_{0}\left(\|-\mu \Delta \mathbf{u}+\nabla p\|_{-1,0}^{2}+\|\nabla \cdot \mathbf{u}+\boldsymbol{\beta} \cdot \nabla p\|^{2}\right) \\
\leq & C_{0}\left(\left\|\mu \nabla^{\perp} w-\mu \nabla q+\nabla p\right\|_{-1,0}^{2}+\mu^{2}\left\|\nabla^{\perp}(w-\nabla \times u)\right\|_{-1,0}^{2}\right. \\
& \left.\quad+\mu^{2}\|\nabla(\nabla \cdot \mathbf{u}-q)\|_{-1,0}^{2}+\|q+\boldsymbol{\beta} \cdot \nabla p\|^{2}\right) \\
\leq & \hat{C}_{0} G_{1}(\mathbf{U}, \mathbf{u}, p ; \mathbf{0}, 0),
\end{aligned}
$$

where $\hat{C}_{0}$ is a constant that depends on $\mu, \boldsymbol{\beta}$, and $\Omega$. Using (3.6), we have

$$
(w, w)=(w-\nabla \times \mathbf{u}, w)+(\nabla \times \mathbf{u}, w) \leq C G_{0}^{1 / 2}(w, \mathbf{u}, q, p)\|w\|,
$$

where $C$ is a constant depending on $\Omega$ and the Poincare constant. Now, cancelling $\|w\|$ on both sides and squaring the remainder, we have

$$
\|w\|^{2} \leq C G_{0}(w, \mathbf{u}, q, p ; \mathbf{0}, 0),
$$


where $C$ is a constant depending on $\Omega$ and the Poincare constant. Now, using (3.6), we have

$$
\begin{aligned}
(q, q) & =(q-\nabla \cdot \mathbf{u}, q)+(\nabla \cdot \mathbf{u}, q) \\
& \leq\|q-\nabla \cdot \mathbf{u}\|\|q\|+\|\nabla \cdot \mathbf{u}\|\|q\| \\
& \leq C G_{0}^{1 / 2}(w, \mathbf{u}, q, p)\|q\|,
\end{aligned}
$$

where $C$ is a constant depending on $\Omega$. Cancelling $\|q\|$ on both sides and squaring the remainder, we have

$$
\|q\| \leq C G_{0}(w, \mathbf{u}, q, p) .
$$

Finally, combining (3.6), (3.8), and (3.10) yields the lower bound. This completes the proof.

3.2. Velocity, flux, and pressure. The first-order least-squares functional corresponding to (2.17) is

$$
\begin{aligned}
G_{1}(\mathbf{U}, \mathbf{u}, p ; \mathbf{f}, g)= & \left\|-\mu(\nabla \cdot \mathbf{U})^{t}+\nabla p-\mathbf{f}\right\|_{-1,0}^{2} \\
& +\|\nabla \cdot \mathbf{u}+\boldsymbol{\beta} \cdot \nabla p-g\|^{2}+\left\|\mathbf{U}-\nabla \mathbf{u}^{t}\right\|^{2}
\end{aligned}
$$

The extended least-squares functional corresponding to (2.18) is

$$
\begin{aligned}
G_{3}(\mathbf{U}, \mathbf{u}, q, p ; \mathbf{f}, g)= & \left\|\mathbf{U}-\nabla \mathbf{u}^{t}\right\|^{2}+\left\|-\mu(\nabla \cdot \mathbf{U})^{t}+\nabla p-\mathbf{f}\right\|^{2}+\|\nabla \times \mathbf{U}\|^{2} \\
& +\|\nabla \cdot \mathbf{u}+\boldsymbol{\beta} \cdot \nabla-g\|^{2}+\|\nabla \operatorname{tr} \mathbf{U}+\nabla(\boldsymbol{\beta} \cdot \nabla p)\|^{2} .
\end{aligned}
$$

Define

$$
\begin{aligned}
M_{1}(\mathbf{U}, \mathbf{u}, \boldsymbol{p}) & =\|\mathbf{U}\|^{2}+\|\mathbf{u}\|_{1}^{2}+\|p\|_{0, \boldsymbol{\beta}}^{2}, \\
M_{2}(\mathbf{U}, \mathbf{u}, \boldsymbol{q}, \boldsymbol{p}) & =\|\mathbf{U}\|_{1}^{2}+\|\mathbf{u}\|_{1}^{2}+\|p\|_{1, \boldsymbol{\beta}}^{2} .
\end{aligned}
$$

Let

$$
\mathbf{V}_{0}=\left\{\mathbf{U} \in H^{1}(\Omega)^{4}: \mathbf{n} \times \mathbf{U}=\mathbf{0} \text { on } \partial \Omega\right\}
$$

Define

$$
\begin{gathered}
\mathscr{V}_{1}=L^{2}(\Omega)^{4} \times H_{0}^{1}(\Omega)^{2} \times Q_{0}(\Omega), \\
\mathscr{V}_{2}=\mathbf{V}_{0} \times H_{0}^{1}(\Omega)^{2} \times Q_{1}(\Omega) .
\end{gathered}
$$

The least-squares variational problem for the compressible Stokes equations corresponding to (2.17) or (2.18) is to minimize the quadratic functional $G_{i}$ over $\mathscr{V}_{i}$ : find $(\mathbf{U}, \mathbf{u}, p) \in \mathscr{V}_{i}$ such that

$$
G_{i}(\mathbf{U}, \mathbf{u}, p ; \mathbf{f}, g)=\inf _{(\mathbf{V}, \mathbf{v}, r) \in \mathscr{V}_{i}} G_{i}(\mathbf{V}, \mathbf{v}, r ; \mathbf{f}, g) \text { for } i=1,2 .
$$


THEOREM 3.2. Under the assumption (2.12), there are two positive constants $c$ and $C$, dependent on $\mu, \boldsymbol{\beta}$, and $\Omega$, such that for all $(\mathbf{U}, \mathbf{u}, p) \in \mathscr{V}_{1}$,

$$
c M_{1}(\mathbf{U}, \mathbf{u}, p) \leq G_{1}(\mathbf{U}, \mathbf{u}, p ; \mathbf{0}, 0) \leq C M_{1}(\mathbf{U}, \mathbf{u}, p) .
$$

Proof. Upper bound in (3.17) is a simple consequence of the triangle inequality and Cauchy-Schwarz inequality. To limit arguments, it is enough to show that lower bound in (3.17) holds for $\widetilde{\mathscr{V}}=H(\operatorname{div} ; \Omega)^{2} \times H_{0}^{1}(\Omega)^{2} \times Q(\Omega)$. Using (2.12) and triangle inequality, we have

$$
\begin{aligned}
& \left\|\nabla \mathbf{u}^{t}\right\|^{2}+\|p\|_{0, \boldsymbol{\beta}}^{2} \\
& \quad \leq C_{0}\left(\|-\mu \Delta \mathbf{u}+\nabla p\|_{-1,0}^{2}+\|\nabla \cdot \mathbf{u}+\boldsymbol{\beta} \cdot \nabla p\|^{2}\right) \\
& \quad \leq C_{0}\left(\left\|-\mu(\nabla \cdot \mathbf{U})^{t}+\nabla p\right\|_{-1,0}^{2}+\mu^{2}\left\|\nabla \cdot\left(\mathbf{U}-\nabla \mathbf{u}^{t}\right)^{t}\right\|_{-1,0}^{2}+\|\nabla \cdot \mathbf{u}+\boldsymbol{\beta} \cdot \nabla p\|^{2}\right) \\
& \quad \leq \hat{C}_{0} G_{1}(\mathbf{U}, \mathbf{u}, p ; \mathbf{0}, 0),
\end{aligned}
$$

where $\hat{C}_{0}$ is a constant that depends on $\mu$ and $\Omega$. Note that

$$
(\mathbf{U}, \mathbf{U})=\left(\mathbf{U}-\nabla \mathbf{u}^{t}, \mathbf{U}\right)+\left(\nabla \mathbf{u}^{t}, \mathbf{U}\right) \leq C\left(\left\|\mathbf{U}-\nabla \mathbf{u}^{t}\right\|\|\mathbf{U}\|+\|\mathbf{u}\|_{1}\|\mathbf{U}\|\right),
$$

where $C$ is a constant depending on $\Omega$. Now cancelling $\|\mathbf{U}\|$ on both sides, squaring the remainder, and using (3.19), we have

$$
\|\mathbf{U}\|^{2} \leq C G_{1}(\mathbf{U}, \mathbf{u}, p ; \mathbf{0}, 0)
$$

where $C$ is a constant depending on $\mu, \boldsymbol{\beta}$, and $\Omega$. Finally, combining (3.19) and (3.20) yields the lower bound. This completes the proof.

The following lemma is basically proved in [5, Lemma 3.2].

LEMMA 3.3. Let $\boldsymbol{\phi}=\left(\phi_{1}, \phi_{2}\right)^{t}$ and $\mathbf{q}=\left(q_{1}, q_{2}\right)^{t}$; if each $q_{i} \in H_{0}^{1}(\Omega) \cap H^{2}(\Omega)$ and each $\phi_{i} \in H^{1}(\Omega)$ is such that $\Delta \phi_{i} \in L^{2}(\Omega)$ and $\mathbf{n} \cdot \nabla \phi_{i}=0$ on $\partial \Omega$, then

$$
|\nabla \cdot \mathbf{q}+\boldsymbol{\beta} \cdot \nabla p|_{1}^{2} \leq C_{\Omega}\left(\left|\nabla \cdot \mathbf{q}+\operatorname{tr} \nabla^{\perp} \boldsymbol{\phi}^{t}+\boldsymbol{\beta} \cdot \nabla p\right|_{1}^{2}+\|\Delta \boldsymbol{\phi}\|^{2}\right) .
$$

Proof. Note that $\operatorname{tr}\left(\nabla^{\perp} \phi_{1}, \nabla^{\perp} \phi_{2}\right)=-\nabla \times \boldsymbol{\phi}$,

$$
\begin{aligned}
|\nabla \cdot \mathbf{q}+\boldsymbol{\beta} \cdot \nabla p|_{1}^{2} & \leq 2\left(|\nabla \cdot \mathbf{q}-\nabla \times \boldsymbol{\phi}+\boldsymbol{\beta} \cdot \nabla p|_{1}^{2}+|\nabla \times \boldsymbol{\phi}|_{1}^{2}\right) \\
& \leq C\left(\left|\nabla \cdot \mathbf{q}+\operatorname{tr} \nabla^{\perp} \boldsymbol{\phi}^{t}+\boldsymbol{\beta} \cdot \nabla p\right|_{1}^{2}+|\boldsymbol{\phi}|_{2}\right) \\
& \leq C\left(\left|\nabla \cdot \mathbf{q}+\operatorname{tr} \nabla^{\perp} \boldsymbol{\phi}^{t}+\boldsymbol{\beta} \cdot \nabla p\right|_{1}^{2}+\|\Delta \boldsymbol{\phi}\|\right),
\end{aligned}
$$

where the constant $C$ depends on $\Omega$.

Due to the above lemma, one may get the following theorem. 
THEOREM 3.4. Under the assumption of (2.12), there are two positive constants $c$ and $C$ dependent on $\mu, \boldsymbol{\beta}$, and $\Omega$ such that for all $(\mathbf{U}, \mathbf{u}, p) \in \mathscr{V}_{2}$,

$$
c M_{2}(\mathbf{U}, \mathbf{u}, q, p) \leq G_{2}(\mathbf{U}, \mathbf{u}, q, p ; \mathbf{0}, 0) \leq C M_{2}(\mathbf{U}, \mathbf{u}, q, p) .
$$

The proof of Theorem 3.4 comes immediately by following techniques similar to those in [5].

4. Finite element approximations. In this section, we provide the finite element approximation of the minimization of the least-squares functionals $G_{0}$ only. Note that an obvious modification in this section also provides the finite element error analysis for the least-squares functionals $G_{1}$ and $G_{2}$. Let $T: H_{0}^{-1}(\Omega)^{2} \rightarrow H_{0}^{1}(\Omega)^{2}$ be the solution operator $(\mathbf{u}=T \mathbf{f})$ for the following elliptic boundary value problem with zero boundary condition $-\Delta \mathbf{u}+\mathbf{u}=\mathbf{f}$ in $\Omega$. It is well known that (see [3, Lemma 2.1])

$$
(\mathbf{f}, T \mathbf{f})=\|\mathbf{f}\|_{-1}^{2}=\sup _{\boldsymbol{\phi} \in H_{0}^{1}(\Omega)^{2}} \frac{(\mathbf{f}, \boldsymbol{\phi})^{2}}{\|\boldsymbol{\phi}\|_{1}^{2}} \quad \forall \mathbf{f} \in H_{0}^{-1}(\Omega)^{2} .
$$

Let $\mathcal{T}_{h}$ be a family of triangulations of $\Omega$ by standard finite element subdivisions of $\Omega$ into quasi-uniform triangles with $h=\max \left\{\operatorname{diam}(K): K \in \mathscr{T}_{h}\right\}$.

Let $\mathscr{V}_{0, h}$ be a finite-dimensional subspace of $\mathscr{V}_{0}$ with an approximation property such that for $(w, \mathbf{u}, q, p) \in \mathscr{V}_{0}$, there exists positive integers $l, m, n \geq 1$ and $s \geq 1$ satisfying

$$
\begin{gathered}
\inf _{w_{h} \in \mathscr{U}_{h}}\left\{\left\|w-w_{h}\right\|+h\left\|w-w_{h}\right\|_{1}\right\} \leq C h^{r}\|w\|_{r}, \\
\inf _{\mathbf{u}_{h} \in \mathscr{T}_{0, h}}\left\{\left\|\mathbf{u}-\mathbf{u}_{h}\right\|+h\left\|\mathbf{u}-\mathbf{u}_{h}\right\|_{1}\right\} \leq C h^{s+1}\|\mathbf{u}\|_{s+1}, \\
\inf _{q_{h} \in \mathscr{U}_{h}}\left\{\left\|q-q_{h}\right\|+h\left\|q-q_{h}\right\|_{1}\right\} \leq C h^{r}\|q\|_{r}, \\
\inf _{p_{h} \in \mathscr{W}_{h}}\left\{\left\|p-p_{h}\right\|+h\left\|p-p_{h}\right\|_{1}\right\} \leq C h^{k+1}\|p\|_{k+1},
\end{gathered}
$$

where $C$ is a positive integer. Then the finite element approximation of (3.4) is to find $\left(w_{h}, \mathbf{u}_{h}, q_{h}, p_{h}\right) \in \mathscr{V}_{0, h}$ which satisfies

$$
G_{0}\left(w_{h}, \mathbf{u}_{h}, q_{h}, p_{h} ; \mathbf{f}, g\right)=\inf _{\left(z_{h}, \mathbf{v}_{h}, r_{h}, s_{h}\right) \in \mathcal{V}_{0, h}} G_{0}\left(z_{h}, \mathbf{v}_{h}, r_{h}, s_{h} ; \mathbf{f}, g\right)
$$

From (4.1), we have

$$
\begin{aligned}
G_{0}(w, \mathbf{u}, q, p ; 0,0)= & \left(T\left(\mu \nabla^{\perp} w-\mu \nabla q+\nabla p\right), \mu \nabla^{\perp} w-\mu \nabla q+\nabla p\right) \\
& +(q+\boldsymbol{\beta} \cdot \nabla p, q+\boldsymbol{\beta} \cdot \nabla p)+(q-\nabla \cdot \mathbf{u}, q-\nabla \cdot \mathbf{u}) \\
& +(w-\nabla \times \mathbf{u}, w-\nabla \times \mathbf{u}) .
\end{aligned}
$$


THEOREM 4.1. Suppose that the assumption in Theorem 3.1 holds. Assume that ( $w, \mathbf{u}$, $q, p) \in \mathscr{V}_{0}$ is the solution of the minimization problem for $G_{1}$ in (3.4) and $\left(w_{h}, \mathbf{u}_{h}, q_{h}, p_{h}\right)$ is the unique minimizer of $G_{0}$ over $\mathscr{V}_{0, h}$. Then

$$
\begin{aligned}
& \left\|w-w_{h}\right\|^{2}+\left\|\mathbf{u}-\mathbf{u}_{h}\right\|_{1}^{2}+\left|q-q_{h}\right|^{2}+\left\|p-p_{h}\right\|_{0, \boldsymbol{\beta}}^{2} \\
& \quad \leq C_{\left(z_{h}, \mathbf{v}_{h}, r_{h}, s_{h}\right) \in V_{0, h}}\left(\left\|w-z_{h}\right\|^{2}+\left\|\mathbf{u}-\mathbf{v}_{h}\right\|_{1}^{2}+\left|q-r_{h}\right|^{2}+\left\|p-s_{h}\right\|_{0, \boldsymbol{\beta}}^{2}\right) .
\end{aligned}
$$

Proof. For convenience, let

$$
\begin{aligned}
{[w, \mathbf{u}, q, p ; z, \mathbf{v}, r, s]=} & \left(T\left(\mu \nabla^{\perp} w-\mu \nabla q+\nabla p\right), \mu \nabla^{\perp} z-\mu \nabla r+\nabla s\right) \\
& +(q+\boldsymbol{\beta} \cdot \nabla p, r+\boldsymbol{\beta} \cdot \nabla s)+(w-\nabla \times \mathbf{u}, z-\nabla \times \mathbf{v}) \\
& +(q-\nabla \cdot \mathbf{u}, r-\nabla \cdot \mathbf{s}) .
\end{aligned}
$$

Then, using (4.1), Theorem 3.1, the orthogonality of the error $\left(w-w_{h}, \mathbf{u}-\mathbf{u}_{h}, q-q_{h}, p-\right.$ $p_{h}$ ) to $\mathscr{V}_{0, h}$, with respect to the above inner product, and the Schwarz inequality, we have the conclusion.

From this theorem and approximate property of $\mathscr{V}_{0, h}$, we have

$$
\begin{aligned}
& \left\|w-w_{h}\right\|^{2}+\left\|\mathbf{u}-\mathbf{u}_{h}\right\|_{1}^{2}+\left\|q-q_{h}\right\|^{2}+\left\|p-p_{h}\right\|_{0, \boldsymbol{\beta}}^{2} \\
& \quad \leq C\left(h^{2 l}\|w\|_{l}^{2}+h^{2 m}\|\mathbf{u}\|_{m+1}^{2}+h^{2 n}\|q\|_{n}^{2}+h^{2 s}\|p\|_{s+1}^{2}\right),
\end{aligned}
$$

where $(w, \mathbf{u}, q, p) \in\left(H^{l}(\Omega) \times H_{0}^{m}(\Omega)^{2} \times H^{n+1}(\Omega)^{2} \times H^{s}(\Omega)\right) \cap \mathscr{V}_{0}$ is the solution of the minimization problem for $G_{0}$ in (3.4) and $\left(w_{h}, \mathbf{u}_{h}, q_{h}, p_{h}\right)$ is the unique minimizer of $G_{0}$ over $\mathscr{V}_{0, h}$.

ACKNOWLEDGMENT. This work was supported by the Korea Research Foundation Grant KRF-2002-070-C00014.

\section{REFERENCES}

[1] P. B. Bochev, Z. Cai, T. A. Manteuffel, and S. F. McCormick, Analysis of velocity-flux first-order system least-squares principles for the Navier-Stokes equations. I, SIAM J. Numer. Anal. 35 (1998), no. 3, 990-1009.

[2] P. B. Bochev and M. D. Gunzburger, Analysis of least squares finite element methods for the Stokes equations, Math. Comp. 63 (1994), no. 208, 479-506.

[3] J. H. Bramble, R. D. Lazarov, and J. E. Pasciak, A least-squares approach based on a discrete minus one inner product for first order systems, Math. Comp. 66 (1997), no. 219, 935-955.

[4] Z. Cai, T. A. Manteuffel, and S. F. McCormick, First-order system least squares for velocityvorticity-pressure form of the Stokes equations, with application to linear elasticity, Electron. Trans. Numer. Anal. 3 (1995), 150-159.

[5]___ First-order system least squares for the Stokes equations, with application to linear elasticity, SIAM J. Numer. Anal. 34 (1997), no. 5, 1727-1741.

[6] Z. Cai and X. Ye, A least-squares finite element approximation for the compressible Stokes equations, Numer. Methods Partial Differential Equations 16 (2000), no. 1, 62-70.

[7] B.-N. Jiang and C. L. Chang, Least-squares finite elements for the Stokes problem, Comput. Methods Appl. Mech. Engrg. 78 (1990), no. 3, 297-311.

[8] R. B. Kellogg and B. Liu, A finite element method for the compressible Stokes equations, SIAM J. Numer. Anal. 33 (1996), no. 2, 780-788. 
[9] J. R. Kweon and R. B. Kellogg, Compressible Navier-Stokes equations in a bounded domain with inflow boundary condition, SIAM J. Math. Anal. 28 (1997), no. 1, 94-108.

[10]___ Compressible Stokes problem on nonconvex polygonal domains, J. Differential Equations 176 (2001), no. 1, 290-314.

Keehwan Kim: Department of Mathematics, Yeungnam University, Kyongsan 712-749, Korea E-mail address: khkim@yu.ac.kr

Sangdong Kim: Department of Mathematics, Kyungpook National University, Daegu 702-701, Korea

E-mail address: skim@knu.ac.kr

Sangsik Shin: Department of Mathematics, Kyungpook National University, Daegu 702-701, Korea

E-mail address: sshin@wmai 1.knu.ac.kr 


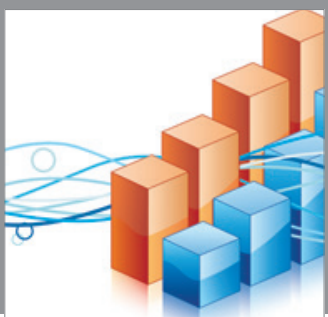

Advances in

Operations Research

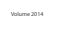

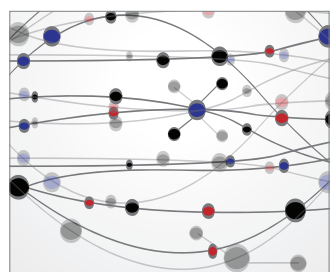

\section{The Scientific} World Journal
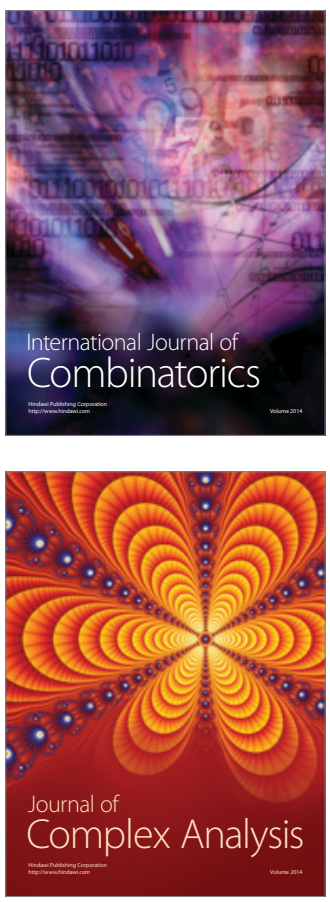

International Journal of

Mathematics and

Mathematical

Sciences
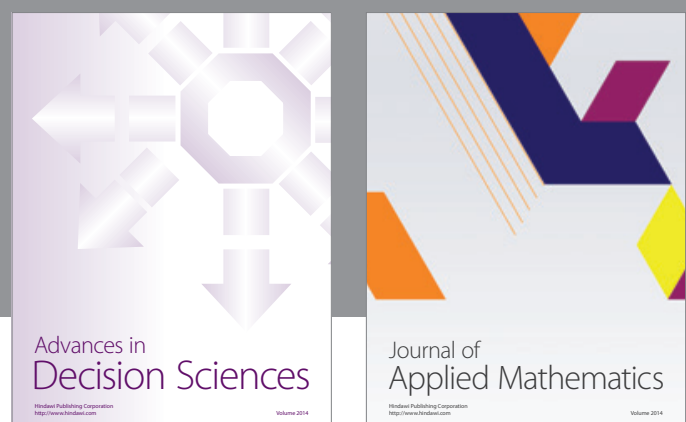

Journal of

Applied Mathematics
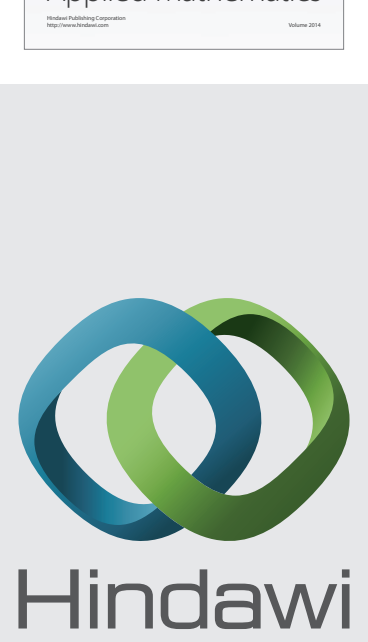

Submit your manuscripts at http://www.hindawi.com
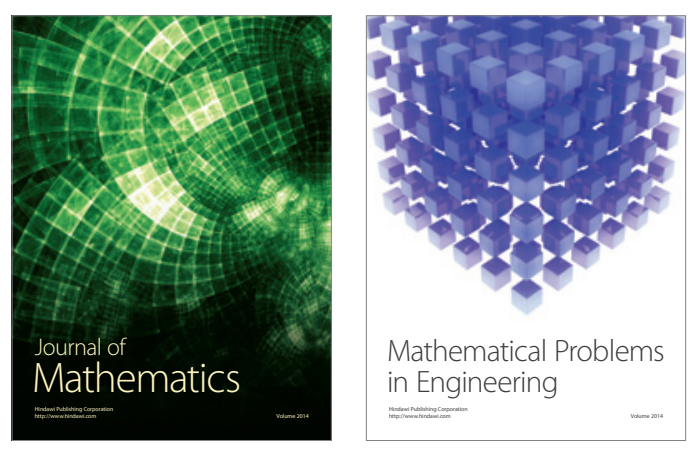

Mathematical Problems in Engineering
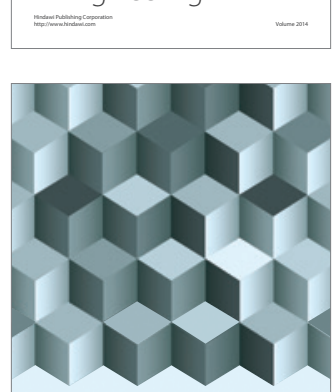

Journal of

Function Spaces
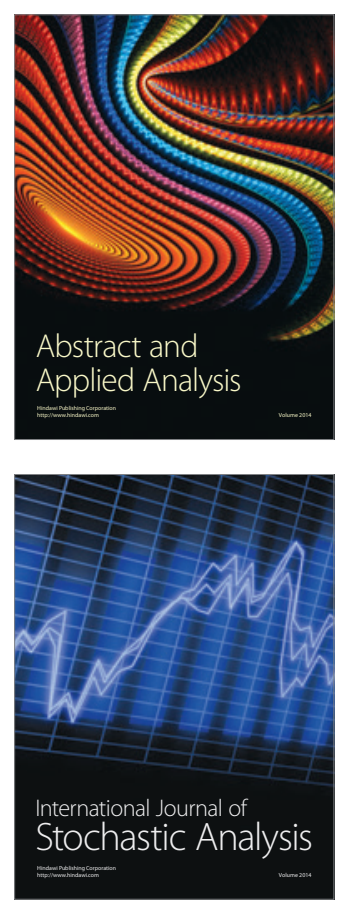

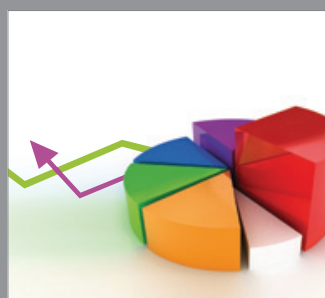

ournal of

Probability and Statistics

Promensencen
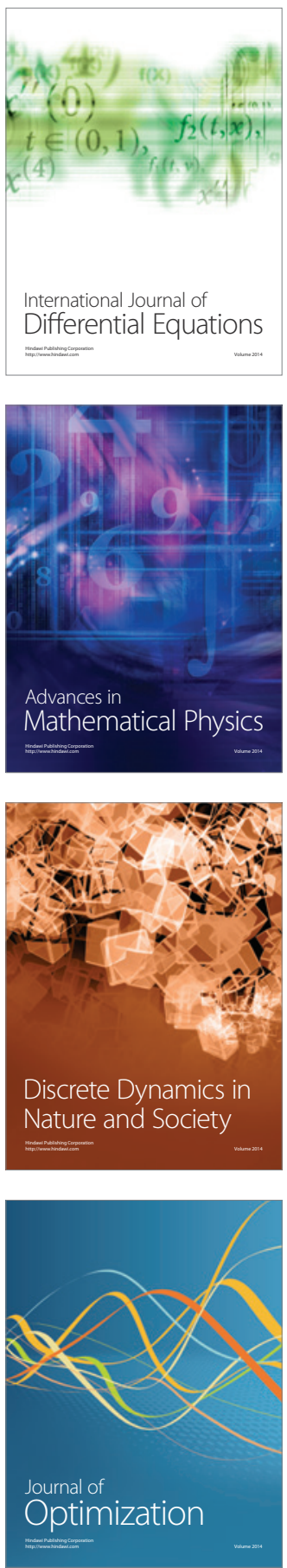